\title{
CONNES EMBEDDABILITY OF GRAPH PRODUCTS
}

\author{
MARTIJN CASPERS
}

Aвstract. We prove that the Connes embedding problem is stable under graph products.

Graph products form a group theoretical construction generalizing free products by adding commutation relations that are dictated by a graph. The construction was first considered by Green in her thesis Gr90 and important examples of graph products arise as right angled Coxeter groups and right angled Artin groups. The formal definition is as follows.

Definition 0.1. Let $\Gamma$ be a graph with vertex set $V \Gamma$ and edge set $E \Gamma$. We may assume that $\Gamma$ has no double edges and no loops, i.e. $(v, v) \notin E \Gamma$. For $v \in V \Gamma$ let $\mathrm{G}_{v}$ be discrete group. Let $\mathrm{G}_{\Gamma}$ be the graph product group which is the discrete group freely generated by $\mathrm{G}_{v}, v \in V \Gamma$ subject to the relation $s t s^{-1} t^{-1}=1$ whenever $s \in \mathrm{G}_{v}$ and $t \in \mathrm{G}_{w}$ with $(v, w) \in E \Gamma$.

Many stability properties of graph products have recently been found by various authors. For example: soficity CHR12, Haagerup property AnDr13 (or CaFi14), residual finiteness Gr90, rapid decay CHR13, linearity HsWi99. and many other properties, see e.g. HeMei95, AnMi11, Chi12.

Also in CaFi14 the operator algebraic graph product was defined. The latter associates to a graph of von Neumann algebras $\mathrm{M}_{v}, v \in V \Gamma$ equipped with faithful normal states a new von Neumann algebra $\mathrm{M}_{\Gamma}$. The construction - generalizing free products - naturally has the property that in case each $\mathrm{M}_{v}$ is the group von Neumann algebra of a discrete group $G_{v}$ then $M_{\Gamma}$ is isomorphic to the group von Neumann algebra of $G_{\Gamma}$. In this paper we show that Connes embeddability for von Neumann algebras is preserved by graph products (see Section 1 for further definitions).

Previous results concerning stability of the Connes embedding problem for free products were found by Popa Pop14 and Isono-Houdayer HoIs15. In Dy08 Brown, Dykema and Jung also found the free entropy dimension of free products. Here we take the approach based on moment formulae that were given by Speicher in Spe93 (see also BoSp94) partly inspired by Junge Jun05] and Nou Nou06 - also proving the result for free products.

Structure. In Section 1 we recall preliminaries on ultraproducts of von Neumann algebras and graph products. Section 2 contains combinatorial arguments to show that graph products of the von Neumann algebras of Voiculescu's free Gaussian functor Voi85 are embeddable. Section 3 proves that graph products preserve the Connes embedding problem.

Acknowledgements. The author thanks Marius Junge for useful discussions and the referee for useful comments and reference Spe93.

\section{Preliminaries}

1.1. Von Neumann algebras. For background on von Neumann algebras we refer to Takesaki's book Tak02. We may and will assume that every von Neumann algebra is represented on its standard GNS space. We shall assume that every von Neumann algebra is separable. Let $\left(\mathcal{M}_{i}, \varphi_{i}\right)_{i \in I}$ be an indexed set of von Neumann algebras $\mathcal{M}_{i}$ with faithful normal states $\varphi_{i}$. Let $\mathcal{U}$ be a free ultrafilter on $\mathcal{I}$. Let $\prod_{i, \mathcal{U}} \mathcal{M}_{i}$ be the Raynaud ultraproduct of von Neumann algebras which can canonically be identified with $\left(\prod_{i, \mathcal{U}}\left(\mathcal{M}_{i}\right)_{*}\right)^{*}$,

Date: February 17, 2020

MC is supported by the grant SFB 878 "Groups, geometry and actions".

MSC2010: 47C15, 46L09, 46L54. 
see Ray02 for details. As in Ray02 we shall write $\left(x_{i}\right)^{\bullet}$ for a bounded sequence $\left(x_{i}\right)_{i \in I}$ with $x_{i} \in \mathcal{M}_{i}$ identified within $\prod_{i, \mathcal{U}} \mathcal{M}_{i}$. Such sequences form a $\sigma$-weakly dense $*$-subalgebra of $\prod_{i, \mathcal{U}} \mathcal{M}_{i}$. We use analogous notation for an ultraproduct of states. Let $e$ be the support projection of the ultraproduct state $\left(\varphi_{i}\right)^{\bullet}$. The ultraproduct von Neumann algebra $\prod_{i, \mathcal{U}}\left[\mathcal{M}_{i}, \varphi_{i}\right]$ is defined as the corner algebra $e\left(\prod_{i, \mathcal{U}} \mathcal{M}_{i}\right) e$. In case each $\left(\mathcal{M}_{i}, \varphi_{i}\right)$ is the hyperfinite $\mathrm{II}_{1}$ factor $\mathcal{R}$ equipped with its unique normal faithful tracial state $\tau$ we set for the resulting algebra $\mathcal{R}^{\mathcal{U}}:=\prod_{i, \mathcal{U}}[\mathcal{R}, \tau]$. We can now state the following conjecture/problem due to A. Connes.

Connes embedding problem: Every separable $\mathrm{II}_{1}$ factor $\mathcal{M}$ embeds into $\mathcal{R}^{\mathcal{U}}$ for some free ultrafilter $\mathcal{U}$.

Here an embedding means that there exists an injective normal $*$-homomorphism $\mathcal{M} \hookrightarrow \mathcal{R}^{\mathcal{U}}$. As the Connes embedding problem is open we shall say that a von Neumann algebra $\mathcal{M}$ is Connes embeddable if an embedding into $\mathcal{R}^{\mathcal{U}}$ exists. We refer to BrOz08, CaLu15, Oza04 for more background and to BCV15] for recent examples of Connes embeddable von Neumann algebras.

Let again $\left(\mathcal{M}_{i}, \varphi_{i}\right)_{i \in I}$ be an indexed set of von Neumann algebras $\mathcal{M}_{i}$ with faithful normal states $\varphi_{i}$. Assume that each $\varphi_{i}$ is tracial. Let $\mathcal{U}$ be a free ultrafilter on $I$. Let $\mathcal{I}_{\mathcal{U}}$ be the $\sigma$-weakly closed ideal in $\prod_{i, \mathcal{U}} \mathcal{M}_{i}$ generated by all elements $\left(x_{i}\right)^{\bullet}$ satisfying $\lim _{i, \mathcal{U}} \tau\left(x_{i}^{*} x_{i}\right)=0$. The Ocneanu ultraproduct $\mathcal{M}^{\mathcal{U}}$ is defined as $\left(\prod_{i, \mathcal{U}} \mathcal{M}_{i}\right) / \mathcal{I}_{\mathcal{U}}$. The Ocneanu ultraproduct $\mathcal{M}^{\mathcal{U}}$ is isomorphic to the ultraproduct $\prod_{i, \mathcal{U}}\left[\mathcal{M}_{i}, \varphi_{i}\right]$, see $[$ AnHa14, Corollary 3.28].

1.2. Graph products. We refer to Gr90 and CaFi14 for the following results. Let $\Gamma$ be a simplicial graph with vertex set $V \Gamma$ and edge set $E \Gamma$. Simplicial means that $\Gamma$ does not have double edges and for every $v \in V \Gamma$ we have $(v, v) \notin E \Gamma$. We presume $\Gamma$ is non-oriented so that $(v, w) \in E \Gamma$ whenever $(w, v) \in E \Gamma$. In this paper we shall assume that $V \Gamma$ is countable so that the graph product of separable von Neumann algebras is again separable. For $v \in V \Gamma$ we set $\operatorname{Link}(v)=\{w \in V \Gamma \mid(w, v) \in E \Gamma\}$. We set $\operatorname{Star}(v)=\operatorname{Link}(v) \cup\{v\}$.

A word is a string of vertices and a word $\mathbf{w}=w_{1} \ldots w_{n}$ is called reduced if the following property holds: if $w_{i}=w_{j}$ with $i<j$ then there exists a $i<k<j$ such that $w_{k} \notin \operatorname{Link}\left(w_{i}\right)$. We let $\mathcal{W}_{\text {red }}$ be the reduced words. We say that two words $\mathbf{v}$ and $\mathbf{w}$ are equivalent if they are equivalent modulo the equivalence relation generated by the two relations:

$$
\begin{array}{rll}
\text { I } \quad\left(v_{1}, \ldots, v_{i}, v_{i+1}, \ldots, v_{n}\right) \simeq\left(v_{1}, \ldots, v_{i}, v_{i+2}, \ldots, v_{n}\right) & \text { if } \quad v_{i}=v_{i+1}, \\
\text { II } \quad\left(v_{1}, \ldots, v_{i}, v_{i+1}, \ldots, v_{n}\right) \simeq\left(v_{1}, \ldots, v_{i+1}, v_{i}, \ldots, v_{n}\right) & \text { if } \quad v_{i} \in \operatorname{Link}\left(v_{i+1}\right) .
\end{array}
$$

Moreover, we say that two words $\mathbf{v}$ and $\mathbf{w}$ are type I equivalent if they are equivalent modulo the sub-equivalence relation generated by relation I. We define the notion type II equivalent in the analogous way.

Every word is equivalent to a reduced word CaFi14, Lemma 1.3]. Out of every equivalence class of words we may therefore pick a single distinguished reduced word, which we call minimal. We let $\mathcal{W}_{\text {min }}$ be the set of minimal words and $\mathcal{W}_{\text {red }}$ be the set of reduced words.

Let $\mathrm{M}_{v}, v \in V \Gamma$ be von Neumann algebras with normal faithful states $\varphi_{v}$. We set $\mathrm{M}_{v}^{\circ}$ for the kernel of $\varphi_{v}$. We define the graph product von Neumann algebra in an explicit way, see CaFi14, Section 2]. Let $\mathcal{H}_{v}$ be the Hilbert space on which $\mathrm{M}_{v}$ acts and let $\xi_{v} \in \mathcal{H}_{v}$ be a distinguished unit vector such that $\varphi_{v}(\cdot)=\left\langle\cdot \xi_{v}, \xi_{v}\right\rangle$. We let $\mathcal{H}_{v}^{\circ}$ be the subspace of $\mathcal{H}_{v}$ consisting of vectors orthogonal to $\xi_{v}$. For a word $\mathbf{v}=v_{1} \ldots v_{n}$ we set $\mathcal{H}_{\mathbf{v}}=\mathcal{H}_{v_{1}}^{\circ} \otimes \ldots \otimes \mathcal{H}_{v_{n}}^{\circ}$. By Lemma CaFi14, Lemma 1.3] we see that if $\mathbf{v} \in \mathcal{W}_{\text {red }}$ is equivalent to $\mathbf{w} \in \mathcal{W}_{\text {red }}$ then there exists a uniquely determined unitary map,

$$
\mathcal{Q}_{\mathbf{v}, \mathbf{w}}: \mathcal{H}_{\mathbf{v}} \rightarrow \mathcal{H}_{\mathbf{w}}: \xi_{v_{1}} \otimes \ldots \otimes \xi_{v_{n}} \mapsto \xi_{v_{\sigma(1)}} \otimes \ldots \otimes \xi_{v_{\sigma(n)}}
$$

where $\sigma$ is as in CaFi14, Lemma $1.3(4)]$. Since every $\mathbf{v} \in \mathcal{W}_{\text {red }}$ has a unique minimal form $\mathbf{v}^{\prime}$ we may simply write $\mathcal{Q}_{\mathbf{v}}$ for $\mathcal{Q}_{\mathbf{v}, \mathbf{v}^{\prime}}$. 
Define the graph product Hilbert space $(\mathcal{H}, \Omega)$ by:

$$
\mathcal{H}=\mathbb{C} \Omega \oplus \bigoplus_{\mathbf{w} \in \mathcal{W}_{\min }} \mathcal{H}_{\mathbf{w}}
$$

Here $\Omega$ is a distinguished unit vector. The graph product state $\varphi_{\Gamma}$ is the vector state given by $\Omega$. For $v \in V \Gamma$, let $\mathcal{W}_{v}$ be the set of minimal reduced words $\mathbf{w}$ such that the concatenation $v \mathbf{w}$ is still reduced and write $\mathcal{W}_{v}^{c}=\mathcal{W}_{\min } \backslash \mathcal{W}_{v}$. Define

$$
\mathcal{H}(v)=\mathbb{C} \Omega \oplus \bigoplus_{\mathbf{w} \in \mathcal{W}_{v}} \mathcal{H}_{\mathbf{w}}
$$

We define the isometry $U_{v}: \mathcal{H}_{v} \otimes \mathcal{H}(v) \rightarrow \mathcal{H}$ in the following way:

$$
\begin{aligned}
& U_{v}: \mathcal{H}_{v} \otimes \mathcal{H}(v) \longrightarrow \mathcal{H} \\
& \xi_{v} \otimes \Omega \stackrel{\simeq}{\longrightarrow} \Omega \\
& \mathcal{H}_{v}^{\circ} \otimes \Omega \stackrel{\simeq}{\longrightarrow} \mathcal{H}_{v}^{\circ} \\
& \xi_{v} \otimes \mathcal{H}_{\mathrm{w}} \stackrel{\simeq}{\longrightarrow} \mathcal{H}_{\mathrm{w}} \\
& \mathcal{H}_{v}^{\circ} \otimes \mathcal{H}_{\mathbf{w}} \stackrel{\simeq}{\longrightarrow} \mathcal{Q}_{v \mathbf{w}}\left(\mathcal{H}_{v}^{\circ} \otimes \mathcal{H}_{\mathbf{w}}\right)
\end{aligned}
$$

Here the actions are understood naturally. Observe that, for any reduced word $\mathbf{w}$, the word $v \mathbf{w}$ is not reduced if and only if $\mathbf{w}$ is equivalent to a reduced word that starts with $v$. It follows that $U_{v}$ is surjective, hence unitary. Define, for $v \in V \Gamma$, the faithful unital normal $*$-homomorphism $\lambda_{v}: \mathcal{B}\left(\mathcal{H}_{v}\right) \rightarrow \mathcal{B}(\mathcal{H})$ by

$$
\lambda_{v}(x)=U_{v}(x \otimes 1) U_{v}^{*} \quad \text { for all } \quad x \in \mathcal{B}\left(\mathcal{H}_{v}\right) .
$$

Observe the $\lambda_{v}$ intertwines the vector states $\omega_{\xi_{v}}$ and $\omega_{\Omega}$. The graph product $\mathrm{M}_{\Gamma}$ is defined as the von Neumann algebra generated by $\cup_{v \in V \Gamma} \lambda_{v}\left(\mathrm{M}_{v}\right)$. We shall identify $\mathrm{M}_{v}$ as a von Neumann subalgebra of $\mathrm{M}_{\Gamma}$ and omit $\lambda_{v}$ in the notation. If each $\mathrm{M}_{v}, v \in V \Gamma$ is a $\mathrm{II}_{1}$-factor, then so is $\mathrm{M}_{\Gamma}$, see CaFi14, Corollary 2.29]. We shall use the fact that $\mathrm{M}_{v}$ and $\mathrm{M}_{w}$ commute whenever $(v, w) \in E \Gamma$ without further reference, see CaFi14, Section 2]. Finally, we mention that graph products satisfy a universal property for which we refer to CaFi14, Proposition $2.22]$.

\section{Graph products and Voiculescu's free Gaussian functor}

In this section we consider graph products of Voiculescu's factors Voi85] (which are in fact free group factors) and find a moment formula.

2.1. Preliminaries on partitions. We let $\mathcal{P}(1, \ldots, n)$ be the set of all partitions of the set $\{1, \ldots, n\}$. We let $\mathcal{P}_{2}(1, \ldots, n)$ be the set of all pair partitions, meaning that every equivalence class consists exactly of 2 elements. In particular the latter set is empty if $n$ is odd. Let $\mathcal{V} \in \mathcal{P}_{2}(1, \ldots, n)$ and write $\mathcal{V}=\left\{\left(e_{1}, z_{1}\right), \ldots,\left(e_{r}, z_{r}\right)\right\}, 2 r=$ $n, e_{i}<z_{i}$. Let $I(\mathcal{V})$ be the set of all pairs $(k, l)$ such that $e_{k}<e_{l}<z_{k}<z_{l}$. Let $\Gamma$ be a simplicial graph and let $\mathbf{v}$ be a (not nessecarily reduced) word of length $n$. We let $\mathcal{P}(\mathbf{v})$ be the set of partitions $\mathcal{V}$ of $(1, \ldots, n)$ with the property that if $k$ and $l$ are equivalent in $\mathcal{V}$ then this implies that $v_{k}=v_{l}$. We let $\mathcal{P}_{2}(\mathbf{v})$ be the subset of $\mathcal{P}(\mathbf{v})$ of pair partitions. For $\mathcal{V} \in \mathcal{P}_{2}(\mathbf{v})$ we define $I_{\Gamma}(\mathcal{V})$ as the subset of $I(\mathcal{V})$ consisting of all pairs $(k, l)$ such that $e_{k}<e_{l}<z_{k}<z_{l}$ and $\left(v_{e_{k}}, v_{e_{l}}\right) \notin E \Gamma$. A pair partition $\mathcal{V}$ is called admissible if $I(\mathcal{V})$ is empty and it is called $\Gamma$-admissible if $I_{\Gamma}(\mathcal{V})$ is empty. We denote $\mathcal{P}_{2, \mathrm{nc}, \Gamma}(\mathbf{v})$ for the subset of $\mathcal{P}_{2}(\mathbf{v})$ consisting of all $\Gamma$-admissible partitions (these can be considered as "non-crossing partitions up to permutations coming from edges of $\left.\Gamma^{\prime \prime}\right)$. Let $\mathcal{V} \in \mathcal{P}(\mathbf{v})$ we say that a sequence of indices $\left(i_{1}, \ldots, i_{n}\right)$ is of class $\mathcal{V}$ if the following property holds: $i_{k}=i_{l}$ and $v_{k}=v_{l}$ if and only if $k$ and $l$ are equivalent in $\mathcal{V}$.

2.2. Voiculescu's free Gaussian functor, graph products and a moment formula. We recall the construction of the factors constructed in Voi85. Let $\mathcal{H}$ be $\mathbb{C}^{2}$ be the two dimensional Hilbert space with orthonormal basis vectors $f_{1}$ and $f_{2}$. Let $\mathcal{K}=\oplus_{n=1}^{\infty} \mathcal{H}^{\otimes n} \oplus \mathbb{C} \cdot \Omega$ where $\Omega$ is a distinguished (vacuum) unit vector. For $f \in \mathcal{H}$ let $a^{*}(f)$ be the creation operator and $a(f)$ be the annihilation operator:

$$
\begin{aligned}
a^{*}(f) \xi & =f \otimes \xi, \quad \xi \in \mathcal{H}^{\otimes n} \\
a(f) \Omega & =0, \\
a(f) g \otimes \xi & =\langle g, f\rangle \xi, \quad \xi \in \mathcal{H}^{\otimes n} .
\end{aligned}
$$


Set,

$$
g_{1}=a^{*}\left(f_{1}\right)+a\left(f_{1}\right), \quad g_{2}=a^{*}\left(f_{2}\right)+a\left(f_{2}\right) .
$$

Let $\mathrm{P}$ be the von Neumann algebra generated by $g_{1}$ and $g_{2}$. In fact $\mathrm{P}$ is equal to the free product of the von Neumann algebras generated by $g_{1}$ and $g_{2}$. Let $\Gamma$ be a simplicial graph. We equip the notions introduced so far in this subsection with a $v \in V \Gamma$ to distinguish them for different vertices. For every $v \in V \Gamma$ let $\mathrm{P}_{v}:=\mathrm{P}$ and let $\mathcal{K}_{v}:=\mathcal{K}$ be the Hilbert space it acts on. For $\mathrm{P}_{v}$ we denote $g_{1, v}$ and $g_{2, v}$ for the generators defined in (2.2). We set $a_{i, v}^{*}$ for $a^{*}\left(f_{i, v}\right)$ as in (2.1). Let $\mathrm{P}_{\Gamma}$ be the graph product von Neumann algebra of the $\mathrm{P}_{v}, v \in V \Gamma$ with graph product state $\tau_{\Gamma}$.

Theorem 2.1. Let $d_{j} \in\left\{g_{i, v} \mid v \in V \Gamma, i \in\{1,2\}\right\}, 1 \leq j \leq n$. Then,

- $\tau_{\Gamma}\left(d_{1} d_{2} \ldots d_{n}\right)$ is 0 in case $n$ is odd.

- Let $v_{j}$ be such that $d_{j}=g_{i, v_{j}}$ for either $i=1$ or $i=2$ and suppose that $n$ is even, say $n=2 r$. Then,

$$
\tau_{\Gamma}\left(d_{1} d_{2} \ldots d_{n}\right)=\# \mathcal{P}_{2, \mathrm{nc}, \Gamma}(\mathbf{v}) \text {. }
$$

Proof. Let $\mathcal{K}_{\Gamma}$ be the graph product Hilbert space on which $\mathrm{P}_{\Gamma}$ acts. Let $\mathcal{W}_{0}$ be the set of all words that are I-equivalent to the words in $\mathcal{W}_{\text {min }}$. For a word $\mathbf{v} \in \mathcal{W}_{0}$ we recall that we have set $\mathcal{H}_{\mathbf{v}}=\mathcal{H}_{v_{1}}^{\circ} \otimes \ldots \otimes \mathcal{H}_{v_{n}}^{\circ}$. We say that the vectors in the latter space have symbol $\mathbf{v}$. We have $\mathcal{K}_{\Gamma} \simeq \oplus_{\mathbf{v} \in \mathcal{W}_{0}} \mathcal{H}_{\mathbf{v}}$ by associativity of tensor products. We describe the actions of the creation and annihilation operators:

$$
a_{i, v_{j}}^{*} \xi_{1} \otimes \ldots \otimes \xi_{n}=\xi_{k_{1}} \otimes \ldots \otimes \xi_{k_{s}} \otimes e_{i, v_{j}} \otimes \xi_{k_{s+1}} \otimes \ldots \otimes \xi_{k_{n}},
$$

where $\xi_{1} \otimes \ldots \otimes \xi_{n}$ has symbol $\mathbf{w} \in \mathcal{W}_{0}$ and $w_{k_{1}} \ldots w_{k_{s}} v_{j} w_{k_{s+1}} \ldots w_{k_{n}}$ is the unique word in $\mathcal{W}_{0}$ that is II-equivalent to $v_{j} w_{1} \ldots w_{n}$ with $w_{k_{s}} \neq v_{j}$ and which satisfies the property that in case $w_{k_{i}}=w_{k_{i+1}}$ we must have $k_{i+1}=k_{i}+1$. Furthermore for a vector $\xi_{1} \otimes \ldots \otimes \xi_{n}$ with symbol $\mathbf{w}$ we find:

$$
a_{i, v_{j}} \xi_{1} \otimes \ldots \otimes \xi_{n}= \begin{cases}\left\langle\xi_{k_{s}}, f_{i}\right\rangle \xi_{k_{1}} \otimes \ldots \otimes \widehat{\xi}_{k_{s}} \otimes \ldots \otimes \xi_{k_{n}} & \text { in case }(*) \text { below holds } \\ 0 & \text { else, }\end{cases}
$$

where $(*)$ means that there exist $k_{1} \ldots k_{n}$ determined by the property that $v_{j} w_{k_{1}} \ldots \widehat{w}_{k_{s}} \ldots w_{k_{n}}$ is II-equivalent to $w_{1} \ldots w_{n}$ (so in particular $w_{k_{s}}=v_{j}$ ), $w_{k_{s-1}} \neq v_{j}, w_{k_{1}} \ldots \widehat{w}_{k_{s}} \ldots w_{k_{n}}$ is in $\mathcal{W}_{0}$ and in case $w_{k_{i}}=w_{k_{i+1}}$ we must have $k_{i+1}=k_{i}+1$.

The action of $d_{1} \ldots d_{n}=\sum_{\left(k_{1}, \ldots, k_{n}\right) \in\{1, *\}^{n}} a_{i_{1}, v_{1}}^{k_{1}} \ldots a_{i_{n}, v_{n}}^{k_{n}}$ on $\Omega$ is thus described by sums of $n$ creation/ annihilation operators. If the trace,

$$
\tau_{\Gamma}\left(a_{i_{1}, v_{1}}^{k_{1}} \ldots a_{i_{n}, v_{n}}^{k_{n}}\right)=\left\langle a_{i_{1}, v_{1}}^{k_{1}} \ldots a_{i_{n}, v_{n}}^{k_{n}} \Omega, \Omega\right\rangle,
$$

is non-zero, then we must have exactly $\frac{n}{2}$ creation operators and $\frac{n}{2}$ annihilation operators occuring in $a_{i_{1}, v_{1}}^{k_{1}} \ldots a_{i_{n}, v_{n}}^{k_{n}}$ and in particular $n$ must be even. This proves the first statement. So let $n$ be even and let $\left(k_{1}, \ldots, k_{n}\right) \in\{1, *\}^{n}$ be such that exactly half of the terms equals 1 and the other half equals $*$. We associate a pair partition to any term $a_{i_{1}, v_{1}}^{k_{1}} \ldots a_{i_{n}, v_{n}}^{k_{n}}$ with non-zero trace in the following way. We connect $s<r$ if $k_{s}=1$ and $k_{r}=*, v_{s}=v_{r}$ and $a_{i_{s}, v_{s}}$ annihilates the vector that was created by $a_{i_{r}, v_{r}}^{*}$. Call the resulting pair partition $\mathcal{V}_{k_{1}, \ldots, k_{n}}$.

Claim: Let $\mathcal{S}$ be the set $\left\{\mathcal{V}_{k_{1}, \ldots, k_{n}} \mid\left(k_{1}, \ldots, k_{n}\right) \in\{1, *\}^{n}\right.$ and $\left.\tau\left(a_{i_{1}, v_{i}}^{k_{1}} \ldots a_{i_{n}, v_{n}}^{k_{n}}\right) \neq 0\right\}$. Then we have $\mathcal{S}=$ $\mathcal{P}_{2, \mathrm{nc}, \Gamma}(\mathbf{v})$.

Proof of the Claim: $\subseteq$. Suppose the inclusion does not hold. Then there exist a partition $\mathcal{V} \in \mathcal{S}$ with $e_{a}<e_{b}<z_{a}<z_{b}$ such that $\left(e_{a}, z_{a}\right) \in \mathcal{V},\left(e_{b}, z_{b}\right) \in \mathcal{V}$ and $\left(v_{a}, v_{b}\right) \notin E \Gamma$. This means that

$$
a_{i_{e_{b}}, v_{e_{b}}}^{k_{e_{b}}}\left(a_{i_{e_{b}}+1, v_{e_{b}+1}}^{k_{e_{e}+1}} \ldots a_{i_{n}, v_{n}}^{k_{n}} \Omega\right)=0
$$

which contradicts that $\mathcal{V} \in \mathcal{S}$.

‥ Again suppose that the inclusion does not hold. Then there exists a pair partition $\mathcal{V} \in \mathcal{P}_{2, n c, \Gamma}$ such that

$$
\tau\left(a_{i_{1}, v_{1}}^{k_{1}} \ldots a_{i_{n}, v_{n}}^{k_{n}}\right)=0,
$$


where the $k_{i}$ are the unique indices determined by:

$$
k_{i}= \begin{cases}1 & \text { if } i=z_{a} \text { for some }\left(e_{a}, z_{a}\right) \in \mathcal{V} \text { with } e_{a}<z_{a} \\ * & \text { if } i=e_{a} \text { for some }\left(e_{a}, z_{a}\right) \in \mathcal{V} \text { with } e_{a}<z_{a}\end{cases}
$$

In particular, $\mathcal{V}=\mathcal{V}_{k_{1}, \ldots, k_{n}}$. But if (2.3) holds and taking into account that $a_{i_{1}, v_{1}}^{k_{1}} \ldots a_{i_{n}, v_{n}}^{k_{n}}$ creates as many vectors as it annihilates (i.e. exactly half of the $k_{i}$ 's equal $*$ ), this shows that we must have

$$
a_{i_{e_{b}}, v_{e_{b}}}^{k_{e_{b}}}\left(a_{i_{e_{b}}+1, v_{e_{b}+1}}^{k_{e_{b}+1}} \ldots a_{i_{n}, v_{n}}^{k_{n}} \Omega\right)=0
$$

for some index $e_{b}$ for which $k_{e_{b}}=1$. This can only happen if there were indices $e_{a}, z_{a}, z_{b}$ such that $e_{a}<e_{b}<$ $z_{a}<z_{b}$ and $\left(e_{a}, z_{a}\right) \in \mathcal{V},\left(e_{b}, z_{b}\right) \in \mathcal{V}$ such that $\left(v_{a}, v_{b}\right) \notin E \Gamma$. This contradicts that $\mathcal{V} \in \mathcal{P}_{2, n c, \Gamma}(\mathbf{v})$.

Remainder of the proof: We now have, putting $2 r=n$,

$$
\begin{aligned}
\tau_{\Gamma}\left(d_{1} \ldots d_{n}\right) & =\sum_{\mathcal{V}=\left\{\left(e_{1}, z_{1}\right), \ldots,\left(e_{r}, z_{r}\right)\right\} \in \mathcal{P}_{2, \mathrm{nc}, \Gamma}(\mathbf{v})}\left\langle a_{i, v_{1}}^{k_{1}} \ldots a_{i_{n}, v_{n}}^{k_{n}} \Omega, \Omega\right\rangle \\
& =\sum_{\substack{\mathcal{V}=\left\{\left(e_{1}, z_{1}\right), \ldots,\left(e_{r}, z_{r}\right)\right\} \in \mathcal{P}_{2, \mathrm{nc}, \Gamma}(\mathbf{v}) \\
k=1}} \prod_{k=1}^{r}\left\langle f_{i_{e_{k}}}, f_{i_{z_{k}}}\right\rangle \\
& =\# \mathcal{P}_{2, \mathrm{nc}, \Gamma}(\mathbf{v}) .
\end{aligned}
$$

This concludes the theorem.

Finally, we recall the following fact (see [Voi85, and CaFi14, Corollary 2.29]).

Theorem 2.2. $\mathrm{P}$ is a $I I_{1}$-factor. In particular $\mathrm{P}_{v}:=\mathrm{P}$ and hence $\mathrm{P}_{\Gamma}$ is a $I I_{1}$-factor.

2.3. The Speicher central limit theorem. We state a version of Speicher's central limit theorem Spe92 adapted to graph products. For a simplicial graph $\Gamma$ and some index set $I$ we let $s: I \times V \Gamma \times I \times V \Gamma \rightarrow\{-1,1\}$ be a $\operatorname{sign}$ function. Let $\mathbf{v}$ be a word with letters in $V \Gamma$ and let $\mathcal{V} \in \mathcal{P}_{2}(\mathbf{v})$. Let $n$ be the length of the word $\mathbf{v}$. We set,

$$
t(\mathcal{V})=\lim _{N \rightarrow \infty} \frac{1}{N^{n / 2}} \sum_{\substack{i_{1}, \ldots, i_{n}=1 \\\left(i_{1}, \ldots, i_{n}\right) \text { is of class } \mathcal{V}}}^{N}\left(\prod_{(a, b) \in I_{\Gamma}(\mathcal{V})} s\left(i_{e_{a}}, v_{e_{a}}, i_{e_{b}}, v_{e_{b}}\right)\right) .
$$

The following theorem is proved in Spe93, Theorem 1] (see also [Spe92, Theorem 1]).

Theorem 2.3. Let $\Gamma$ be a simplicial graph. Let $\mathcal{A}$ be a $*$-algebra generated by self-adjoint elements $b_{i, v}, i \in$ $\mathbb{N}, v \in V \Gamma$. Presume that there is a sign function $s: \mathbb{N} \times V \Gamma \times \mathbb{N} \times V \Gamma \rightarrow\{-1,1\}$ satisfying $s(i, v, j, w)=1$ whenever $(v, w) \in E \Gamma$ such that:

For $v \in V \Gamma$ and $N \in \mathbb{N}$ set

$$
b_{i, v} b_{j, w}=s(i, v, j, w) b_{j, w} b_{i, v}
$$

$$
S_{N, v}:=\frac{b_{1, v}+\ldots+b_{N, v}}{\sqrt{N}} .
$$

Assume that for every (not necessarily reduced) word $\mathbf{v}=v_{1} \ldots v_{n}$ the quantity $t(\mathcal{V})$ exists for every $\mathcal{V} \in \mathcal{P}_{2}(\mathbf{v})$. Then there exists a state $\rho$ on $\mathcal{A}$ that satisfies the following:

$$
\lim _{N \rightarrow \infty} \varphi\left(S_{N, v_{1}} \ldots S_{N, v_{n}}\right)= \begin{cases}0 & n \text { is odd } \\ \sum_{\mathcal{V} \in \mathcal{P}_{2}(\mathbf{v}), \mathcal{V}=\left\{\left(e_{1}, z_{1}\right), \ldots,\left(e_{r}, z_{r}\right)\right\}} t(\mathcal{V}) & n=2 r\end{cases}
$$

$\rho\left(b_{i, v}^{2}\right)=1$ and $\rho\left(b_{i, v} b_{j, w}\right)=0$ in case $(i, v) \neq(j, w)$.

Next we show that $t(\mathcal{V})$ in the previous theorem can be computed almost everywhere. In order to do so, let $\geq$ be some linear order on $\mathbb{N} \times V \Gamma$. Naturally the symbol $>$ stands for $\geq$ but not equal.

Lemma 2.4. For $\Gamma$ a simplicial graph, let $\mathbf{s}=(s(v, i, w, j))_{v, w \in V \Gamma, i, j \in \mathbb{N}}$ be an infinite random matrix with the properties: 
(1) $s(i, v, j, w)=s(j, w, i, v)$ for every $i, j \in I$ and $v, w \in V \Gamma$,

(2) $s(i, v, j, w)=1$ whenever $(v, w) \in E \Gamma$,

(3) $s(i, v, j, w)$ with $(i, v)>(j, w)$ are independent,

(4) $\operatorname{prob}(s(i, v, j, w)=1)=p$ and $\operatorname{prob}(s(i, v, j, w)=-1)=q:=1-p$, whenever $(v, w) \notin E \Gamma$.

Let $\mathbf{v}$ be a (not necessarily reduced) word with letters in $V \Gamma$. Then, for almost every $\mathbf{s}$ we have for all $\mathcal{V} \in \mathcal{P}_{2}(\mathbf{v})$ that

$$
t(\mathcal{V})=(p-q)^{\# I_{\Gamma}(\mathcal{V})} .
$$

Proof. As $s(i, v, j, w)=1$ whenever $(v, w) \in E \Gamma$ we have (trivially) that $\operatorname{prob}(s(i, v, j, w)=1)=1$ whenever $(v, w) \in E \Gamma$. So the lemma follows from [Spe93, Theorem 2].

2.4. Matrix models. We now prove that the von Neumann algebra $P_{\Gamma}$ introduced in Section 2.2 is embeddable. For the moment assume that $\Gamma$ is a finite graph. Let $N \in \mathbb{N}$ and set $I_{N}=\{0, \ldots, N\}$. Let $s: I_{N} \times V \Gamma \times I_{N} \times V \Gamma \rightarrow\{-1,1\}$ be a sign function satisfying the properties:

(1) $s(i, v, j, w)=s(j, w, i, v)$;

(2) $s(i, v, i, v)=-1$.

We let $x_{i, v}$ be algebraic generators of an algebra $\mathcal{A}$ satisfying the following relations:

$$
x_{i, v} x_{j, w}-s(i, v, j, w) x_{j, w} x_{i, v}=2 \delta_{i, j} \delta_{v, w} .
$$

In particular $x_{i, v}^{2}=1$ and it follows from these (anti-)commutation relations that $\mathcal{A}$ is finite dimensional. Fix a linear order on $I_{N} \times V \Gamma$. For $A \subseteq I_{N} \times V \Gamma$ we set,

$$
x_{A}:=\prod_{(i, v) \in A} x_{i, v}
$$

where the product is taken with respect to the linear order. The sets $x_{A}$ form a basis of $\mathcal{A}$. We equip $\mathcal{A}$ with the $*$-structure given by $x_{i, v}^{*}=x_{i, v}$. Let $\varphi$ be the tracial function $\mathcal{A} \rightarrow \mathbb{C}$ defined by $\varphi\left(x_{A}\right)=\delta_{A, \emptyset}$. Then $\langle x, y\rangle=\varphi\left(y^{*} x\right)$ defines an inner product and hence a Hilbert space $L^{2}(\mathcal{A}, \varphi)$. We define partial isometries $a_{i, v}^{*}$ and $a_{i, v}$ by

$$
a_{i, v}^{*} x_{A}= \begin{cases}x_{i, v} X_{A} & \text { if }(i, v) \notin A, \\ 0 & \text { if }(i, v) \in A,\end{cases}
$$

and

$$
a_{i, v} x_{A}= \begin{cases}x_{i, v} X_{A} & \text { if }(i, v) \in A, \\ 0 & \text { if }(i, v) \notin A,\end{cases}
$$

Note that (2.6) is the adjoint of (2.7). Then we set

$$
b_{i, v}=a_{i, v}^{*}+a_{i, v} .
$$

These operators satisfy the relations

$$
b_{i, v} b_{j, w}=s(i, v, j, w) b_{j, w} b_{i, v}
$$

We set for $N \in \mathbb{N}$ even,

$$
S_{N, v, 1}=\frac{1}{\sqrt{N}} \sum_{i=0}^{N-1} b_{2 i, v}, \quad S_{N, v, 2}=\frac{1}{\sqrt{N}} \sum_{i=0}^{N-1} b_{2 i+1, v},
$$

Now we assume that $s: I_{\infty} \times V \Gamma \times I_{\infty} \times V \Gamma \rightarrow\{-1,1\}$ is an infinite random matrix with entries being independent identically distributed random variables subject to conditions (11) and (2) and such that whenever $(v, w) \notin E \Gamma$ we have $\operatorname{prob}(s(i, v, j, w)=1)=\operatorname{prob}(s(i, v, j, w)=-1)=\frac{1}{2}$. The following result is now a direct consequence of Theorem 2.1, Theorem 2.3 and Lemma 2.4. Note that Theorem 2.3 shows that there is some $\rho$ satisfying the moment formula of Theorem 2.3 , but in fact the extra condition $b_{i, v}^{2}=1$ implies that $\rho$ is $\varphi$ above (see Theorem 3 of Spe93 and in particular the discussion after it). 
Theorem 2.5. Let $\Gamma$ be a finite simplicial graph and let $V \Gamma=\left\{v_{1}, \ldots, v_{n}\right\}$. For any $*$-polynomial $Q$ in $2 \# V \Gamma$ non-commutating variables we have

$$
\tau\left(Q\left(g_{1, v_{1}}, \ldots, g_{1, v_{n}}, g_{2, v_{1}}, \ldots, g_{2, v_{n}}\right)\right)=\lim _{N \rightarrow \infty} \varphi\left(Q\left(S_{N, v_{1}, 1}, \ldots, S_{N, v_{n}, 1}, S_{N, v_{1}, 2}, \ldots, S_{N, v_{n}, 2}\right)\right)
$$

for almost every $s$.

Let $\mathcal{U}$ be a free ultrafilter on $\mathbb{N}$. We wish to prove that $\mathrm{P}_{\Gamma}$ (see Section 2.2) is Connes embeddable by defining an injective $*$-homomorphism $\Phi$ by setting

$$
\Phi\left(Q\left(g_{1, v_{1}}, \ldots, g_{1, v_{n}}, g_{2, v_{1}}, \ldots, g_{2, v_{n}}\right)\right)=\prod_{N, \mathcal{U}} Q\left(S_{N, v_{1}, 1}, \ldots, S_{N, v_{n}, 1}, S_{N, v_{1}, 2}, \ldots, S_{N, v_{n}, 2}\right) .
$$

However, the entries of the ultra product on the right hand side may not be bounded. Therefore we need bounded cut-offs and at this point the argument is exactly the same as in Nou06. Section 3]. Meaning, let $C$ be any constant such that $\left\|g_{1, v_{1}}\right\| \leq C$. Let $\chi_{[-C, C]}$ be the characteristic function of the interval $[-C, C]$. We set $\widetilde{S}_{N, v_{i}, i}:=\chi_{[-C, C]}\left(S_{N, v, i}\right) S_{N, v, i}$. What is proved in Nou06, Section 3] is that (2.8) also holds if $S_{N, v_{i}, i}$ is replaced by $\widetilde{S}_{N, v_{i}, i}$. As a consequnce:

Theorem 2.6. The graph product von Neumann algebra $\mathrm{P}_{\Gamma}$ is Connes embeddable.

\section{EMBEDdABILITY OF GRAPH PRODUCTS}

We prove the main result of this paper, namely that the Connes embedding problem is stable under graph products. The following Lemma 3.1] is proved in the first paragraph of the proof of [Jun05, Lemma 7.18].

Lemma 3.1. Let $\mathrm{N}$ and $\mathrm{M}$ be von Neumann algebras. Let $\mathcal{E}: \mathrm{N} \rightarrow \mathrm{M}$ be a conditional expectation. Let $\omega$ be a normal state on $\mathrm{M}$. Let $f \in \mathrm{N}$ be the support of $\omega \circ \mathcal{E}$ and let $e \in \mathrm{M}$ be the support of $\omega$. Then $f$ commutes with every element in eMe.

Lemma 3.2. Let $\mathrm{M}_{v}, v \in V \Gamma$ be von Neumann algebras with normal faithful tracial state $\tau_{v}$. Suppose that for every $v \in V \Gamma$ there are von Neumann algebras $\mathrm{A}_{v, i}$ with normal faithful tracial states $\tau_{v, i}$ with a trace preserving embedding:

$$
\pi_{v}: \mathrm{M}_{v} \rightarrow \prod_{i, \mathcal{U}}\left[\mathrm{A}_{v, i}, \tau_{v, i}\right]
$$

then there exists a trace preserving embedding,

$$
\pi_{\Gamma}: \mathrm{M}_{\Gamma} \rightarrow \prod_{i, \mathcal{U}}\left[\mathrm{A}_{\Gamma, i}, \tau_{\Gamma, i}\right]
$$

Proof. Let $j_{v, i}: \mathrm{M}_{v, i} \rightarrow \mathrm{M}_{\Gamma, i}$ be the natural trace preserving embedding. The predual maps $\left(j_{v, i}\right)_{*}:\left(\mathrm{M}_{\Gamma, i}\right)_{*} \rightarrow$ $\left(\mathrm{M}_{v, i}\right)_{*}$ are contractive and hence induce a mapping in the ultraproduct $\left(j_{v}\right)_{*}: \prod_{i, \mathcal{U}}\left(\mathrm{M}_{\Gamma, i}\right)_{*} \rightarrow \prod_{i, \mathcal{U}}\left(\mathrm{M}_{v, i}\right)_{*}$. We let

$$
j_{v}: \prod_{i, \mathcal{U}} \mathrm{M}_{v, i} \rightarrow \prod_{i, \mathcal{U}} \mathrm{M}_{\Gamma, i}
$$

be the dual of this mapping. Note that $\prod_{i, \mathcal{U}} \mathrm{M}_{v, i}$ is the dual of $\prod_{i, \mathcal{U}}\left(\mathrm{M}_{v, i}\right)_{*}$ via the pairing $\left\langle\left(x_{i}\right)^{\bullet},\left(\omega_{i}\right)^{\bullet}\right\rangle=$ $\lim _{i, \mathcal{U}} \omega_{i}\left(x_{i}\right)$ and therefore explicitly $j_{v}\left(\left(x_{i}\right)^{\bullet}\right)=\left(j_{v, i}\left(x_{i}\right)\right)^{\bullet}$.

Let $e_{v}, v \in V \Gamma$ be the support projection of the ultraproduct state $\left(\tau_{v, i}\right)^{\bullet}$. Let $f$ be the support projection of the ultraproduct state $\left(\tau_{\Gamma, i}\right)^{\bullet}$. Note that $j_{v}$ identifies $\prod_{i, \mathcal{U}} \mathrm{M}_{v, i}$ as a subalgebra of $\prod_{i, \mathcal{U}} \mathrm{M}_{\Gamma, i}$ and $\left(\tau_{\Gamma, i}\right)^{\bullet}$ restricts to $\left(\tau_{v, i}\right)^{\bullet}$ CaFi14.

Recall that $e_{v} \prod_{i, \mathcal{U}} \mathrm{M}_{v, i} e_{v}=\prod_{i, \mathcal{U}}\left[\mathrm{M}_{v, i}, \tau_{v, i}\right]$ and similarly $f \prod_{i, \mathcal{U}} \mathrm{M}_{\Gamma, i} f=\prod_{i, \mathcal{U}}\left[\mathrm{M}_{\Gamma, i}, \tau_{\Gamma, i}\right]$. Set $\rho_{v}$ : $\prod_{i, \mathcal{U}}\left[\mathrm{M}_{v, i}, \tau_{v, i}\right] \rightarrow \prod_{i, \mathcal{U}}\left[\mathrm{M}_{\Gamma, i}, \tau_{\Gamma, i}\right]$ by defining $\rho_{v}\left(e_{v} x e_{v}\right)=f j_{v}\left(e_{v} x e_{v}\right) f$, where $x \in \prod_{i, \mathcal{U}} \mathrm{M}_{v, i}$. By Lemma $3.1 f$ commutes with the image of $j_{v}$, from which we conclude that $\rho_{v}$ is a $*$-homomorphism. Set $\alpha_{v}=\rho_{v} \circ \pi_{v}$. Note that $\alpha_{v}$ is faithful: indeed let $0 \leq x \in \mathrm{M}_{v}$ be non-zero. Then $\left(\tau_{\Gamma, i}\right)^{\bullet}\left(\alpha_{v}(x)\right)=\tau_{v}(x) \neq 0$. Hence $\alpha_{v}(x) \neq 0$. 
We shall now verify the universal property CaFi14, Proposition 2.22]. Let $(v, w) \in E \Gamma$. For $x=\left(x_{i}\right)^{\bullet}$ and $y=\left(y_{i}\right)^{\bullet}$ in respectively $\prod_{i, \mathcal{U}} \mathrm{M}_{v, i}$ and $\prod_{i, \mathcal{U}} \mathrm{M}_{w, i}$ we have since $x_{i} y_{i}=y_{i} x_{i}$ :

$$
\begin{aligned}
& \rho_{v}\left(e_{v} x e_{v}\right) \rho_{v^{\prime}}\left(e_{v^{\prime}} x e_{v^{\prime}}\right)=f j_{v}\left(e_{v} x e_{v}\right) f j_{w}\left(e_{w} y e_{w}\right) f=f j_{v}\left(e_{v} x e_{v}\right) j_{w}\left(e_{w} y e_{w}\right) f \\
= & f\left(j_{v, i}\left(e_{v, i} x_{i} e_{v, i}\right)\right)^{\bullet}\left(j_{w, i}\left(e_{w, i} y_{i} e_{w, i}\right)\right)^{\bullet} f=f\left(j_{w, i}\left(e_{w, i} y_{i} e_{w, i}\right)\right)^{\bullet}\left(j_{v, i}\left(e_{v, i} x_{i} e_{v, i}\right)\right)^{\bullet} f,
\end{aligned}
$$

and the latter expression equals $\rho_{v}\left(e_{v} x e_{v}\right) \rho_{v^{\prime}}\left(e_{v^{\prime}} x e_{v^{\prime}}\right)$. So the images of $\rho_{v}$ and $\rho_{w}$ commute and hence the images of $\alpha_{v}$ and $\alpha_{w}$ commute.

Next, let $\mathbf{v}=v_{1} \ldots v_{n}$ be a reduced word. For $1 \leq k \leq n$ let $a_{k} \in \mathrm{M}_{v_{k}}^{\circ}$. Since $e_{v_{k}}\left(\prod_{i, \mathcal{U}} \mathrm{A}_{v_{k}, i}\right) e_{v_{k}}$ equals $\prod_{i, \mathcal{U}}\left[\mathrm{A}_{v_{k}, i}, \tau_{v_{k}, i}\right]$ we may approximate $\pi_{v_{k}}\left(a_{k}\right) \in \prod_{i, \mathcal{U}}\left[\mathrm{A}_{v_{k}, i}, \tau_{v_{k}, i}\right]$ in the strong topology with a bounded net $\left(a_{k, i, s}\right)_{s \in S}^{\bullet}$ where $a_{k, i, s} \in \mathrm{A}_{v_{k}, i}$ (by Kaplansky's density theorem). Since $\pi_{v_{k}}$ is trace preserving it follows that $\lim _{s \in S} \lim _{i, \mathcal{U}} \tau_{v_{k}, i}\left(a_{k, i, s}\right)=0$. Therefore we may replace $a_{k, i, s}$ by $a_{k, i, s}^{\circ}:=a_{k, i, s}-\tau_{v_{k}, i}\left(a_{k, i, s}\right) \in \mathrm{A}_{v_{k}, i}^{\circ}$ and still have $\left(a_{k, i, s}^{\circ}\right)_{s \in S}^{\bullet} \rightarrow \pi_{v_{k}}\left(a_{k}\right)$ strongly. Then,

$$
\begin{aligned}
& \tau_{\Gamma}\left(\alpha_{v_{1}}\left(a_{1}\right) \ldots \alpha_{v_{n}}\left(a_{n}\right)\right) \\
= & \lim _{s \in S} \tau_{\Gamma}\left(f\left(j_{v_{1}, i}\left(a_{1, i, s}^{\circ}\right)\right)^{\bullet} f \alpha_{v_{2}}\left(a_{2}\right) \ldots \alpha_{v_{n}}\left(a_{n}\right)\right) \\
= & \lim _{s_{1} \in S_{1}} \ldots \lim _{s_{n} \in S_{n}} \tau_{\Gamma}\left(f\left(j_{v_{1}, i}\left(a_{1, i, s_{1}}^{\circ}\right)\right)^{\bullet} f \ldots f\left(j_{v_{n}, i}\left(a_{n, i, s_{n}}^{\circ}\right)\right)^{\bullet} f\right) \\
= & \lim _{s_{1} \in S_{1}} \ldots \lim _{s_{n} \in S_{n}} \lim _{i, \mathcal{U}} \tau_{\Gamma, i}\left(j_{v_{1}, i}\left(a_{1, i, s_{1}}^{\circ}\right) \ldots j_{v_{n}, i}\left(a_{n, i, s_{n}}^{\circ}\right)\right),
\end{aligned}
$$

which equals zero as $\tau_{\Gamma, i}\left(j_{v_{1}, i}\left(a_{1, i, s_{1}}^{\circ}\right) \ldots j_{v_{n}, i}\left(a_{n, i, s_{n}}^{\circ}\right)\right)=0$. Hence we may apply CaFi14, Proposition 2.22] which concludes that there exists a trace preserving embedding $\pi_{\Gamma}: \mathrm{M}_{\Gamma} \rightarrow \prod_{i, \mathcal{U}}\left[\mathrm{A}_{\Gamma, i}, \tau_{\Gamma, i}\right]$.

Lemma 3.3. Let $\mathrm{M}$ be a type $I_{1}$ factor with normal faithful tracial state $\tau$. Consider $M_{n}(\mathbb{C})$ with normalized trace. There exists a trace preserving embedding $\varphi_{n}: M_{n}(\mathbb{C}) \rightarrow \mathrm{M}$.

Proof. Let $p_{1}, \ldots, p_{n}$ be $n$ mutually orthogonal projections in $\mathrm{M}$ with $\tau\left(p_{n}\right)=n^{-1}$. Since $\mathcal{M}$ is a type II factor let $u_{i, j}, i \neq j$ be partial isometries with $u_{i, j} u_{i, j}^{*}=p_{i}, u_{i, j}^{*} u_{i, j}=p_{j}$. Put $u_{i, i}=p_{i}$. Let $e_{i, j}$ be the matrix units of $M_{n}(\mathbb{C})$. Then extending $\varphi_{n}: e_{i, j} \mapsto u_{i, j}$ linearly gives the required mapping.

Theorem 3.4. Let $\Gamma$ be a countable simplicial graph. For every $v \in V \Gamma$ let $\mathrm{M}_{v}$ be a $I I_{1}$ factor with normal faithful tracial state $\tau_{v}$. The graph product $\mathrm{M}_{\Gamma}$ is Connes embeddable if and only if for every $v \in V \Gamma, \mathrm{M}_{v}$ is Connes embeddable.

Proof. Assume that $\Gamma$ is finite. The only if part is trivial as $\mathrm{M}_{v}$ is a subalgebra of $\mathrm{M}_{\Gamma}$. Conversely, for every $v \in V \Gamma$ let $\pi_{v}: \mathrm{M}_{v} \rightarrow \prod_{i, \mathcal{U}}\left[\mathrm{A}_{v, i}, \tau_{v, i}\right]$ be an embedding into an ultraproduct of matrix algebras $\mathrm{A}_{v, i}$ with tracial states $\tau_{v, i}$. Lemma 3.2 yields an embedding $\mathrm{M}_{\Gamma} \rightarrow \prod_{i, \mathcal{U}}\left[\mathrm{A}_{\Gamma, i}, \tau_{\Gamma, i}\right]$. Let $\mathrm{P}_{v, i}$ be a copy of $\mathrm{P}$ with vacuum state $\tau_{v, i} . \mathrm{P}_{v, i}$ is a $\mathrm{II}_{1}$-factor by Theorem 2.2. Let $\mathrm{P}_{\Gamma, i}$ be the graph product of these factors. By Lemma 3.3 each $\mathrm{A}_{v, i}$ is an expected subalgebra of $\mathrm{P}_{v, i}$ and so $\mathrm{A}_{\Gamma, i}$ is an expected subalgebra of $\mathrm{P}_{\Gamma, i}$. Hence we must prove that $\prod_{i, \mathcal{U}}\left[\mathrm{P}_{\Gamma, i}, \tau_{\Gamma, i}\right]$ is Connes embeddable. In turn by Jun05, Lemma 7.14] it suffices to prove that each $\mathrm{P}_{\Gamma, i}$ is Connes embeddable. The latter is Theorem 2.6. For infinite $\Gamma$ the result follows from an inductive limit argument, c.f. the proof of [CaFi14, Corollary 2.17].

\section{REFERENCES}

[AnHa14] H. Ando, U. Haagerup, Ultraproducts of von Neumann algebras, J. Funct. Anal. 266 (2014), 6842-6913.

[AnDr13] Y. Antolin, D. Dreesen, The Haagerup property is stable under graph products, arXiv:1305.6748

[AnMi11] Y. Antolin, A. Minasyan, Tits alternatives for graph products, J. Reine Angew. Math. (to appear), arXiv:1111.2448 [Bau74] H. Bauer, Wahrscheinlichkeitstheorie und Grundzüge der Masstheorie, 2nd edn. Berlin: Walter de Gruyter 1974.

[BCV15] M. Brannan, B. Collins, R. Vergnioux, The Connes embedding property for quantum group von Neumann algebras, Trans. Amer. Math. Soc. (to appear), arXiv: 1412.7788.

[BoSp94] M. Bozejko, R. Speicher, Completely positive maps on Coxeter groups, deformed commutation relations, and operator spaces, Math. Ann. 300 (1994), no. 1, 97-120.

[BrOz08] N. Brown, N. Ozawa, $C^{*}$-algebras and finite-dimensional approximations, Graduate Studies in Mathematics, 88. American Mathematical Society, Providence, RI, 2008. xvi+509 pp. 
[CaFi14] M. Caspers, P. Fima, Graph products of operator algebras, arXiv: 1411.2799.

[Chi12] I. M. Chiswell, Ordering graph products of groups, Internat. J. Algebra Comput., 22 (2012), no. 4, 1250037, 14 pp.

[CHR12] L. Ciobanu, D. Holt and S. Rees, Sofic groups: graph products and graphs of groups, Pacific J. Math. 271 (2014), No. $1,53-64$.

[CHR13] L. Ciobanu, F. Holt, S. Rees, Rapid decay is preserved by graph products, J. Topol. Anal. 5 (2013), no. 2, $225-237$.

[Dy08] K. Dykema, N. Brown, K. Jung, Free entropy dimension in amalgamated free products, Proc. London Math. Soc. 97 (2008), 339-367.

[Gr90] E.R. Green, Graph products, PhD thesis, University of Leeds, 1990, http://ethesis.whiterose.ac.uk/236

[HeMei95] S. Hermiller, J. Meier, Algorithms and geometry for graph products of groups, J. Algebra 171 (1995), no. 1, 230-257.

[Hia01] F. Hiai, q-deformed Araki-Woods algebras, Operator Algebras and Mathematical Physics (Constanta, 2001), Theta, Bucharest, 2003, pp. 169-202.

[HoIs15] C. Houdayer, Y. Isono, Free independence in ultraproduct von Neumann algebras and applications, J. London Math. Soc. (to appear).

[HsWi99] T. Hsu, T. Wise, On linear and residual properties of graph products, Michigan Math. J. 46 (1999) 251-259.

[Jun05] M. Junge, Embedding of the operator space OH and the logarithmic 'little Grothendieck inequality', Invent. Math. 161 (2005), no. 2, 225-286.

[CaLu15] V. Capraro, M. Lupini, Introduction to sofic and hyperlinear groups and Connes' embedding conjecture, Lecture Notes in Mathematics, vol. 2136.

[Nou06] A. Nou, Asymptotic matricial models and QWEP property for $q$-ArakiWoods algebras, J. Funct. Anal. 232 (2006), no. $2,295-327$.

[Oza04] N. Ozawa, About the QWEP conjecture, Internat. J. Math. 15 (2004), 501-530.

[Pop14] S. Popa, Independence properties in subalgebras of ultraproduct $\mathrm{II}_{1}$ factors, J. Funct. Anal. 266 (2014), $5818-5846$.

[Ray02] Y. Raynaud, On ultrapowers of non-commutative Lp-spaces, J. Operator Theory 48 (2002), $41-68$.

[Spe92] R. Speicher, A noncommutative central limit theorem, Math. Z. 209 (1992), no. 1, 55-66.

[Spe93] R. Speicher, Generalized statistics of macroscopic fields, Lett. Math. Phys. 27 (1993), no. 2, 97-104.

[Tak02] M. Takesaki, Theory of operator algebras I, Encyclopaedia of Mathematical Sciences, 124. Operator Algebras and Noncommutative Geometry, 5. Springer-Verlag, Berlin, 2002. xx+415 pp

[Voi85] D.V. Voiculescu, Symmetries of some reduced free product $C^{*}$-algebras, Letcure Notes in Math. 1132 (1985), 556-588.

M. Caspers, Fachbereich Mathematik und Informatik der Universität Münster, Einsteinstrasse 62,48149 Münster, GERMANy

E-mail address: martijn.caspers@uni-muenster.de 